\title{
Article
}

\section{Nasal Morphology and Its Correlation to Craniofacial Morphology in Lateral Cephalometric Analysis}

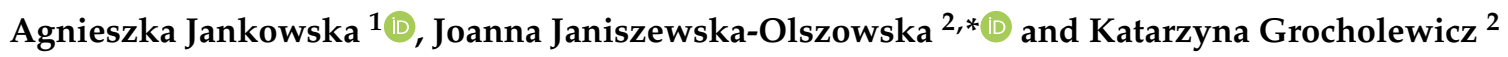 \\ 1 Private Practice “Dental Clinic Jankowscy", 68-200 Żary, Poland; agnieszkajankowska2301@gmail.com \\ 2 Department of Interdisciplinary Dentistry, Pomeranian Medical University in Szczecin, \\ 70-111 Szczecin, Poland; katgro@pum.edu.pl \\ * Correspondence: jjo@pum.edu.pl; Tel.: +48-91-466-1690
}

Citation: Jankowska, A.;

Janiszewska-Olszowska, J.;

Grocholewicz, K. Nasal Morphology

and Its Correlation to Craniofacial

Morphology in Lateral Cephalometric

Analysis. Int. J. Environ. Res. Public

Health 2021, 18, 3064. https://

doi.org/10.3390/ijerph18063064

Academic Editor: Gianrico Spagnuolo

Received: 23 February 2021

Accepted: 11 March 2021

Published: 16 March 2021

Publisher's Note: MDPI stays neutral with regard to jurisdictional claims in published maps and institutional affiliations.

Copyright: (c) 2021 by the authors. Licensee MDPI, Basel, Switzerland. This article is an open access article distributed under the terms and conditions of the Creative Commons Attribution (CC BY) license (https:// creativecommons.org/licenses/by/ $4.0 /)$.

\begin{abstract}
Nose shape, size, and inclination influence facial appearance, but few studies concern the relationship between the nasal profile and craniofacial structures. The objective of this study was to analyze association of nasal cephalometric variables with skeletal structures, age, and sex. Cephalometric and nasal analysis was performed in 386 Polish orthodontic patients (aged 9-25 years). Student $t$-test and Mann-Whitney test were used to compare quantitative variables and Pearson's or Spearman's correlation coefficients—to find correlations. Soft tissue facial convexity angle correlates to Holdaway ratio, ANB (A-Nasion-B), and Wits appraisal. Nasal dorsum axis, nose length, nose depth (1) and nose depth (2), nose hump, lower dorsum convexity, and columella convexity increase with age. Nasal base angle, nasolabial angle, nasomental angle, soft tissue facial convexity and nasal bone angle decrease with age. Nasal base angle and nasomental angle are smaller in females. Thus, a relationship exists between nasal morphology and sagittal jaw configuration. Nasal parameters significantly change with age. Sexual dimorphism characterizes nasal bone angle and nasomental angle.
\end{abstract}

Keywords: nose; nose profile; cephalometry; orthodontics

\section{Introduction}

The nose constitutes the most prominent part of the profile and occupies the most visible position in the face. It has a great influence on facial appearance and profile [1-5]. Thus, nasal balance and harmony are very important. Noses come in various sizes and shapes: upturned or straight, with or without a hump. Similarly, as a facial profile, the nasal dorsum can be classified as: straight, convex, or concave [6].

The nose, lips, and chin create facial harmony. The ideal nasal proportion requires a straight nasal dorsum with the dorsal cartilage and nasal tip cartilage above the nasal tip, forming the supratip break, and the alar rims 1-2 mm superior to the columella in the lateral view $[2,4,5,7]$. The main difference between an attractive and a below-average woman's face is not in the proportion qualities of the nose, but in the relationship of the nasal and craniofacial measurements [5]. Moreover, the concept of an ideal nose is different among races, sexes, and ethnic groups [1,4]. Typical racial and ethnic differences in nasal morphology refer to the width, protrusion, and inclination of the longitudinal axis of the nostrils [5].

The nose shape in the cephalometric soft tissue profile is described by the nasolabial angle (NLA). By some authors, it is considered an excellent clinical and cephalometric parameter to reveal the anteroposterior position of the maxilla $[1,8]$. The NLA has two components: the inclination of the upper lip (lower nasolabial angle) and the upward nasal tip inclination (upper nasolabial angle) [1,9]. The inclination of the upper lip has a strong correlation with the amount of retraction of upper incisors, but nasal tip inclination has no correlation with incisor retraction $[1,9,10]$. Lo and Hunter [9] divided the NLA angle into its two contributing angles. (1) Nasal upward tip angle-the angle formed when the posterior 
columella point $(\mathrm{PCm})$ (the most posterior point of the lower border of the nose) tangent is extended anteriorly to intersect with the Frankfurt horizontal plane/lower border of the nose to the Frankfurt horizontal plane; (2) the upper lip inclination - the angle formed by the PCm-Ls (labrale superius) line with the Frankfurt horizontal plane/inclination of the upper lip to the Frankfurt horizontal plane [1].

Previous studies evaluated the relationship between the nasal upward tip angle and vertical maxillary skeletal pattern. It was found that if an adult patient presents with an upturned nose, it might indicate that the maxillary plane is tipped anticlockwise [1]. Robinson et al. [6] analyzed lateral radiographs and proved that nasal shape followed the underlying skeletal pattern. Contrary results were obtained by Fitzgerald et al. [10], who found no correlation between soft tissue and skeletal measurements in the wellbalanced profile.

Morphology of the nose shows a correlation with skeletal Classes. A pronounced elevation of the nasal dorsum and projection of the nasal bone is found in Class II subjects [6,11-14]. Class III subjects tend to have a concave dorsum and Class I subjects-a straight dorsum of the nose [12]. No relationship was found between the amount of nasal development and skeletal Class, the growth of the nose observed was relatively independent of the underlying skeletal hard tissue (skeletal Classes) [15].

Previous investigators stated that shape and size of the nose, and its inclination, have a significant influence on the orthodontic treatments plan. Excessive nasal growth in conjunction with extractions would probably have resulted in additional lip flattening and contributed directly to a poorly balanced profile [14]. Thus, orthodontic diagnosis and treatment planning should include nose evaluation and prediction of changes in facial aesthetics in a cumulative effect of the growth, development, and treatment [16].

Few studies have been found concerning the relationship between nasal profile and craniofacial structures. Thus, the purpose of this study was to analyze (1) correlations between nasal parameters and craniofacial skeletal structures; (2) correlations of nasal parameters with age (growth); and (3) sexual dimorphism of nasal parameters.

\section{Materials and Methods}

This study has been exempt from ethical approval, based on a written reply from the ethical commission. Sample size has been verified using an online power and sample size calculator (statisticalsolutions.net) assuming power of 0.8 , level of significance at 0.05 , and effect size equal to that calculated from a subsample of 100 patients.

After obtaining permission, 386 cephalograms of orthodontic patients aged 9-25 years were selected from the records of the Department of Radiology Pomeranian Medical University in Szczecin, based on the following criteria:

- Caucasian origin;

- $\quad$ Age 9-25 years;

- Good visibility of all cephalometric and nasal structures;

- Natural head position, the teeth occluded in the maximum intercuspation, relaxed lips;

- No craniofacial deformities;

- No fixed braces present at the time of the cephalogram.

Cephalometric analysis, according to Segner and Hasund [17], was performed by the first author using a specialized computer software (Ortodoncja 8.0, Ortobajt, Wrocław, Poland). Analysis of nasal morphology was made according to Gulsen et al. [4] on acetate paper using a $0.5-\mathrm{mm}$ pencil. The nasal and cephalometric landmarks used are presented in Figure 1 and described in Table 1. All cephalometric variables used are listed and described in Table 2. 


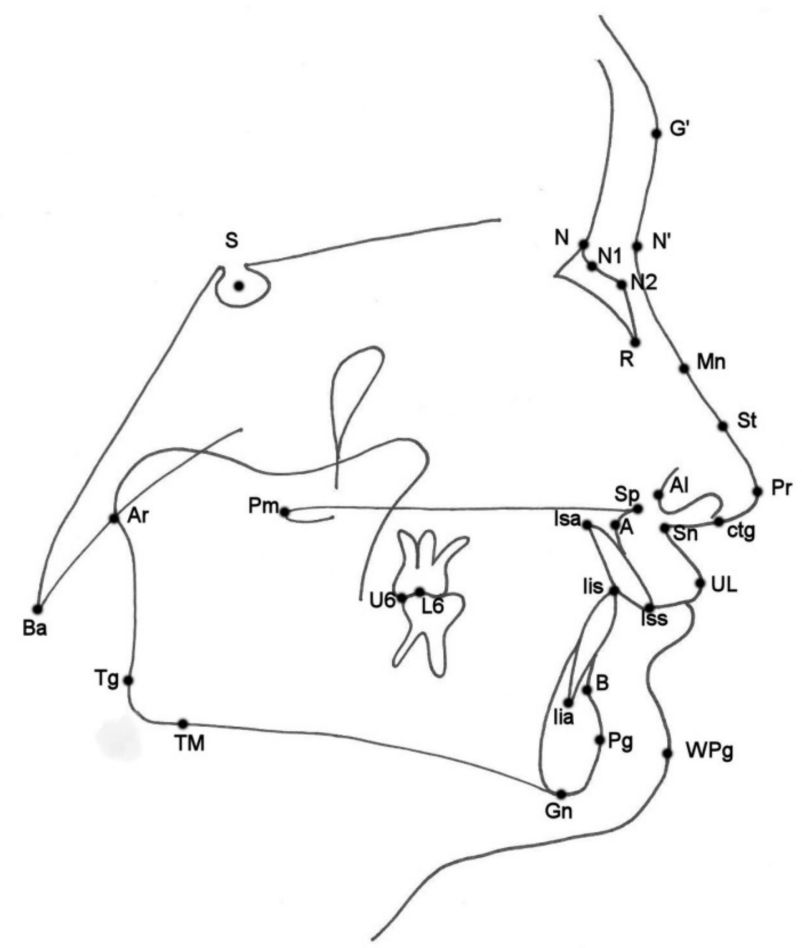

Figure 1. Nasal and cephalometric landmarks.

Table 1. Nasal and cephalometric landmarks.

\begin{tabular}{|c|c|c|}
\hline & Landmark & Definition \\
\hline $\mathrm{G}^{\prime}$ & Soft tissue glabella & $\begin{array}{c}\text { Soft tissue point on the inferior part of the forehead } \\
\text { between the eyebrows }\end{array}$ \\
\hline $\mathrm{N}^{\prime}$ & Soft tissue nasion & $\begin{array}{l}\text { Soft tissue point-borderline between the forehead and } \\
\text { the nose }\end{array}$ \\
\hline $\mathrm{Mn}$ & Midnasale & $\begin{array}{l}\text { Soft tissue point in the middle of the distance between } \\
\text { the points } N^{\prime} \text { and } \operatorname{Pr}\end{array}$ \\
\hline St & Supratip & $\begin{array}{c}\text { Soft tissue point halfway between the Midnasale point } \\
\text { and the Pronasale points }\end{array}$ \\
\hline $\operatorname{Pr}$ & Pronasale & Tip of the nose (soft tissue point) \\
\hline Ctg & Columella & $\begin{array}{c}\text { The most prominent point on the Sn-Pr curve, } \\
\text { borderline between lower part of the nose contour and } \\
\text { nasal tip }\end{array}$ \\
\hline Sn & Subnasale & Soft tissue point between columella and upper lip. \\
\hline UL & Upper Lip & The most protruding point of the upper lip \\
\hline Al. & Alare & $\begin{array}{c}\text { The most receding soft tissue point of the nasal } \\
\text { alar curvature. }\end{array}$ \\
\hline WPg & Pogonion & The most prominent point on the soft tissue chin \\
\hline $\mathrm{N}^{\circ}$ & Nasion & $\begin{array}{l}\text { The most receding point of the anterior surface of the } \\
\text { frontonasal suture }\end{array}$ \\
\hline N1 & Nasion 1 & $\begin{array}{l}\text { The most receding point of the frontal curvature on the } \\
\text { nasal bone }\end{array}$ \\
\hline N2 & Nasion 2 & $\begin{array}{l}\text { The most protruding point of the frontal curvature on } \\
\text { the nasal bone }\end{array}$ \\
\hline $\mathrm{R}$ & Rhinion & The most prominent and inferior point on the nasal bone \\
\hline $\mathrm{S}$ & Sella & Centre the sella turcica \\
\hline Sp & Spina nasalis anterior & The most protruding point of the nasal spine \\
\hline A & Subspinale & $\begin{array}{l}\text { The most posterior point on the anterior surface of } \\
\text { the maxilla }\end{array}$ \\
\hline Iss & Incision superius & Incisal edge of the most protruded upper central incisor \\
\hline
\end{tabular}


Table 1. Cont.

\begin{tabular}{ccc}
\hline & Landmark & Definition \\
\hline Isa & Incision superius apicalis & Apex of upper central incisor \\
B & Supramentale & $\begin{array}{c}\text { The most posterior point on the anterior surface of the } \\
\text { mandible }\end{array}$ \\
Iis & Incision infernus & Incisal edge of the most protruded lower central incisor \\
Apgex of lower central incisor
\end{tabular}

Table 2. Cephalometric and nasal variables.

\begin{tabular}{|c|c|c|c|c|}
\hline & Abbreviation (unit) & Name & Definition & Interpretation \\
\hline \multirow{12}{*}{ 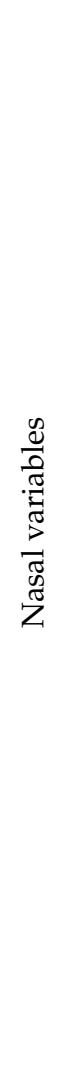 } & $\mathrm{N}^{\prime}-\mathrm{St}(\mathrm{mm})$ & The axis of dorsum & $\begin{array}{l}\text { Distance between the soft tissue } \\
\text { nasion point and the supratip point }\end{array}$ & Length of the nasal dorsum \\
\hline & $\mathrm{N}^{\prime}-\operatorname{Pr}(\mathrm{mm})$ & Nasal length & $\begin{array}{c}\text { Distance between the } \mathrm{N}^{\prime} \text { point and } \\
\text { the Pr point }\end{array}$ & Total nasal length \\
\hline & Nose depth (1) (mm) & Nose depth (1) & $\begin{array}{l}\text { Perpendicular distance between Pr } \\
\text { and the } N^{\prime} \text {-Sn line }\end{array}$ & $\begin{array}{l}\text { Sagittal position of the nose } \\
\text { tip referring to the face }\end{array}$ \\
\hline & $\mathrm{Al}-\operatorname{Pr}(\mathrm{mm})$ & Nose depth (2) & Distance between points $\mathrm{Al}$ and $\operatorname{Pr}$ & $\begin{array}{l}\text { Sagittal position of the nose } \\
\text { tip referring to alar base }\end{array}$ \\
\hline & Hump (mm) & Hump & $\begin{array}{l}\text { Perpendicular distance between the } \\
\text { axis of dorsum and its most } \\
\text { prominent soft tissue point }\end{array}$ & Convexity of nasal dorsum \\
\hline & NBA $\left(^{\circ}\right)$ & Nasal base angle & $\begin{array}{l}\text { Angle between the } G^{\prime}-S n \text { line and } \\
\text { the long axis of the nostril }\end{array}$ & $\begin{array}{l}\text { Inclination of nasal base } \\
\text { referring to the face }\end{array}$ \\
\hline & $\operatorname{NMA}\left({ }^{\circ}\right)$ & Nasomental angle & $\begin{array}{l}\text { Angle between the axis of the } \\
\text { dorsum and the Pr-WPg line }\end{array}$ & $\begin{array}{l}\text { Relation between nasal } \\
\text { dorsum inclination and chin } \\
\text { position }\end{array}$ \\
\hline & $\operatorname{SFC}\left(^{\circ}\right)$ & $\begin{array}{l}\text { Soft tissue facial } \\
\text { convexity }\end{array}$ & $\begin{array}{l}\text { Angle between the lines } G^{\prime}-S n \text { and } \\
\text { Sn-WPg line }\end{array}$ & Profile convexity \\
\hline & Dconv (mm) & $\begin{array}{l}\text { Lower dorsum } \\
\text { convexity }\end{array}$ & $\begin{array}{c}\text { Perpendicular distance between the } \\
\text { Mn-Pr line and its most prominent } \\
\text { point }\end{array}$ & $\begin{array}{c}\text { Convexity of the lower part } \\
\text { of nasal dorsum }\end{array}$ \\
\hline & Cconv (mm) & Columella convexity & $\begin{array}{l}\text { Perpendicular distance between the } \\
\text { Pr-Sn line and the most anterior } \\
\text { point on the convexity of columella }\end{array}$ & Convexity of nasal base \\
\hline & NboneL (mm) & Nasal bone length & $\begin{array}{l}\text { The line constructed between the } \mathrm{N} \\
\text { point and the } \mathrm{R} \text { point }\end{array}$ & $\begin{array}{l}\text { Length of long axis of nasal } \\
\text { bone }\end{array}$ \\
\hline & NboneA $\left({ }^{\circ}\right)$ & Nasal bone angle & $\begin{array}{l}\text { The posterior angle between the } \\
\text { lines N1-N2 and N2-R }\end{array}$ & Curvature of the nasal bone \\
\hline
\end{tabular}


Table 2. Cont

\begin{tabular}{|c|c|c|c|c|}
\hline & Abbreviation (unit) & Name & Definition & Interpretation \\
\hline \multirow{19}{*}{ 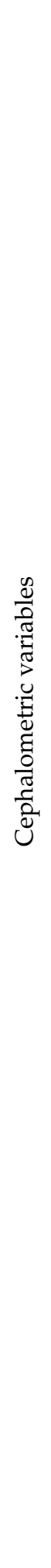 } & SNA $\left(^{\circ}\right)$ & $\begin{array}{l}\text { Sella-Nasion to point } \\
\text { A angle }\end{array}$ & $\begin{array}{l}\text { Angle between Sella-Nasion line } \\
\text { and the Nasion-A line }\end{array}$ & $\begin{array}{l}\text { Sagittal position of the } \\
\text { maxillary alveolar process } \\
\text { referring to anterior } \\
\text { cranial fossa }\end{array}$ \\
\hline & SNB $\left(^{\circ}\right)$ & $\begin{array}{l}\text { Sella-Nasion to point } \\
\text { B angle }\end{array}$ & $\begin{array}{l}\text { Angle between lines Sella-Nasion } \\
\text { and Nasion-B }\end{array}$ & $\begin{array}{l}\text { Sagittal position the alveolar } \\
\text { part of the mandible } \\
\text { referring to anterior } \\
\text { cranial fossa }\end{array}$ \\
\hline & $\operatorname{ANB}\left(^{\circ}\right)$ & A point to $B$ point angle & Difference between SNA and SNB & $\begin{array}{l}\text { Sagittal relation between } \\
\text { maxilla and mandible }\end{array}$ \\
\hline & $\operatorname{SNPg}\left({ }^{\circ}\right)$ & $\begin{array}{l}\text { Sella-Nasion to point } \\
\text { Pg angle }\end{array}$ & $\begin{array}{l}\text { Angle between the lines } \\
\text { Sella-Nasion and Nasion-Pg }\end{array}$ & $\begin{array}{l}\text { Sagittal position of the chin } \\
\text { referring to anterior } \\
\text { cranial fossa }\end{array}$ \\
\hline & NSBa $\left(^{\circ}\right)$ & Cranial base angle & $\begin{array}{l}\text { Angle between the lines } \\
\text { Nasion-Sella and Sella- Basion }\end{array}$ & $\begin{array}{l}\text { Inclination of clivus referring } \\
\text { to anterior cranial fossa }\end{array}$ \\
\hline & Gn-tgo-Ar $\left(^{\circ}\right)$ & Mandibular angle & $\begin{array}{l}\text { Angle between the lines Gn-tgo and } \\
\text { tgo-Ar }\end{array}$ & $\begin{array}{l}\text { Angulation between } \\
\text { mandibular corpus } \\
\text { and ramus }\end{array}$ \\
\hline & NL-NSL $\left(^{\circ}\right)$ & Maxillary base angle & $\begin{array}{c}\text { The angle between the NL } \\
\text { (maxillary base) line and } \\
\text { Nasion-Sella line }\end{array}$ & $\begin{array}{l}\text { Maxillary inclination to the } \\
\text { anterior cranial fossa }\end{array}$ \\
\hline & ML-NSL $\left(^{\circ}\right)$ & Mandibular base angle & $\begin{array}{l}\text { Angle between the ML (mandibular } \\
\text { base) line and Nasion-Sella line }\end{array}$ & $\begin{array}{l}\text { Inclination of mandibular } \\
\text { base to the anterior } \\
\text { cranial fossa }\end{array}$ \\
\hline & ML-NL $\left(^{\circ}\right)$ & Intermaxillary angle & $\begin{array}{l}\text { Angle between the ML (mandibular) } \\
\text { line and NL (maxillary) line }\end{array}$ & $\begin{array}{l}\text { Inclination between the bases } \\
\text { of maxilla and mandible }\end{array}$ \\
\hline & $\operatorname{NLA}\left({ }^{\circ}\right)$ & Nasolabial angle & $\begin{array}{c}\text { Angle between the points ctg, } \\
\text { Sn, UL }\end{array}$ & $\begin{array}{l}\text { Relationship between the } \\
\text { upper lip and columella }\end{array}$ \\
\hline & $\mathrm{H}$ & Holdaway ratio & $\begin{array}{l}\text { Angle between the lines UL-WPg } \\
\text { and NB }\end{array}$ & $\begin{array}{l}\text { Relationship between soft } \\
\text { tissue profile and hard } \\
\text { tissue profile }\end{array}$ \\
\hline & $1+1-\left(^{\circ}\right)$ & Interincisal angle & $\begin{array}{l}\text { Angle between the axes of upper } \\
\text { and lower incisors }\end{array}$ & $\begin{array}{l}\text { Inclination of upper and } \\
\text { lower central incisors }\end{array}$ \\
\hline & $1+: \mathrm{NA}\left({ }^{\circ}\right)$ & Upper incisor angle & $\begin{array}{l}\text { Angle between long axis of the most } \\
\text { protruded upper central incisor and } \\
\text { NA line }\end{array}$ & $\begin{array}{l}\text { Upper central incisor } \\
\text { inclination to maxillary base }\end{array}$ \\
\hline & $1-: \mathrm{NB}\left(^{\circ}\right)$ & Lower incisor angle & $\begin{array}{l}\text { Angle between long axis of the most } \\
\text { protruded lower central upper } \\
\text { incisor and NB line }\end{array}$ & $\begin{array}{l}\text { Lower central incisor } \\
\text { inclination to } \\
\text { mandibular base }\end{array}$ \\
\hline & Pg:NB (mm) & Distance Pg-NB & $\begin{array}{l}\text { Distance between Pg point and } \\
\text { NB line }\end{array}$ & $\begin{array}{l}\text { Sagittal position of the chin } \\
\text { referring to alveolar part of } \\
\text { the mandible }\end{array}$ \\
\hline & $1+\mathrm{NA}(\mathrm{mm})$ & Upper incisor distance & $\begin{array}{l}\text { Distance from upper incisor to } \\
\text { NA line }\end{array}$ & $\begin{array}{l}\text { Sagittal position of upper } \\
\text { incisal edge to maxilla }\end{array}$ \\
\hline & $1-\mathrm{NB}(\mathrm{mm})$ & Lower incisor distance & $\begin{array}{l}\text { Distance from upper incisor to } \\
\text { NB line }\end{array}$ & $\begin{array}{l}\text { Sagittal position of lower } \\
\text { incisal edge to mandible }\end{array}$ \\
\hline & Index $(\%)$ & $\begin{array}{l}\text { Lower face height } \\
\text { index }\end{array}$ & $\begin{array}{l}\text { Ratio between the middle and lower } \\
\text { face heights }\end{array}$ & $\begin{array}{l}\text { Lower face height in } \\
\text { proportion to midface }\end{array}$ \\
\hline & Wits (mm) & Wits appraisal & $\begin{array}{l}\text { Distance between the perpendicular } \\
\text { projections of points A and B on the } \\
\text { occlusal plane }\end{array}$ & $\begin{array}{l}\text { Sagittal relation between } \\
\text { maxilla and mandible }\end{array}$ \\
\hline
\end{tabular}

Cephalometric and nasal analysis was repeated six months later, by the same investigator, in 100 randomly selected cephalograms. Repeatability of measurements was assessed using one-sided Wilcoxon test. The level of clinical significance has been set at 5 degrees for angular measurements and $2 \mathrm{~mm}$ for linear measurements.

Statistical analysis was performed using R software, version 4.0.3 [18]. Data distribution normality was assessed using Shapiro-Wilk test. The level of significance was estab- 
lished at $p=0.05$. Comparisons of quantitative variables between the groups were made using Student $t$-test (for data of normal distribution) or Mann-Whitney test (otherwise). Correlations between quantitative variables were assessed using Pearson's correlation coefficient (for data of normal distribution) or Spearman's correlation coefficient (otherwise). The power of correlation was assessed according to the following schema [19]:

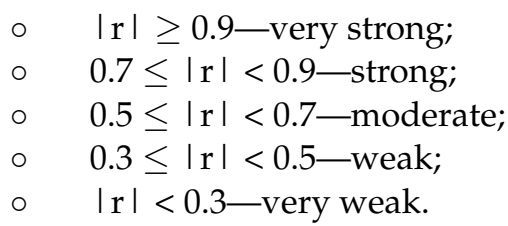

\section{Results}

Sample size verification revealed that for the correlation coefficient between the SFC (Soft tissue facial convexity) angle and the $\mathrm{H}$ (Holdaway ratio) angle (0.763) a sample size of 11 subjects would be sufficient to show the significance, for the correlation coefficient between the NMA (nasomental angle) angle and the $\mathrm{H}$ angle $(-0.517)$ the sample size yielded 37 and for the correlation coefficient between the NLA angle and the ANB (a point to B point angle) angle (0.247) 327 subjects would be sufficient to show the significance.

Wilcoxon one-sided test for repeated measurements revealed no differences between repeated measurements exceeding the level of clinical significance ( 5 degrees for angular measurements and $2 \mathrm{~mm}$ for linear measurements) for hump, NBA (nasal base angle), NMA, SFC. For N'-St (the axis of dorsum) and $N^{\prime}-\operatorname{Pr}$ (nasal length) it was $1 \%$, for nose depth (1) and NBoneA (nasal bone angle) it was $2 \%$, for nose depth (2): $3 \%$. For $1+$ :NA $\left(^{\circ}\right)$ (upper incisor angle) and 1-:NB $\left(^{\circ}\right)$ (Lower incisor angle) it was $4 \%$, for $1+: \mathrm{NA}(\mathrm{mm})$ and NBoneL (nasal bone length) it was 5\%. For Pg:NB [mm] it was $6 \%$. The highest percentage of discordant measurements $(11 \%)$ was noted for the position of the lower incisors $(1-: \mathrm{NB})(\mathrm{mm})$.

Characteristics of the study group, according to age, sex, and skeletal Class are presented in Table 3. Analysis of the data distribution normality are presented in Table 4. Cephalometric and nasal values of the study group are presented in Table 5.

Table 3. Characteristics of the study group $(n=386)$.

\begin{tabular}{|c|c|c|}
\hline & & Values \\
\hline Age & $\begin{array}{c}\text { Mean } \pm \text { SD } \\
\text { median } \\
\text { quartiles }\end{array}$ & $\begin{array}{c}14.19 \pm 3.58 \\
14 \\
12-16\end{array}$ \\
\hline Sex & $\begin{array}{l}\text { Females } \\
\text { Males }\end{array}$ & $\begin{array}{l}229(59.33 \%) \\
157(40.67 \%)\end{array}$ \\
\hline Skeletal Class & $\begin{array}{c}\text { I } \\
\text { II } \\
\text { III }\end{array}$ & $\begin{array}{c}173(44.82 \%) \\
138(35.75 \%) \\
75(19.43 \%)\end{array}$ \\
\hline
\end{tabular}

Table 4. Data normality (Shapiro-Wilk test, $p<0.05$ ).

\begin{tabular}{ccc}
\hline Variable & $p$-Value & Normality \\
\hline Age & $<0.001$ & No \\
SNA $\left(^{\circ}\right)$ & 0.006 & No \\
SNB $\left(^{\circ}\right)$ & 0.001 & No \\
ANB $\left(^{\circ}\right)$ & $<0.001$ & No \\
SNPg $\left(^{\circ}\right)$ & 0.001 & No \\
NSBa $\left({ }^{\circ}\right)$ & 0.516 & Yes \\
GntgoAr $\left(^{\circ}\right)$ & 0.332 & Yes \\
NL-NSL $\left(^{\circ}\right)$ & $<0.001$ & No \\
\hline
\end{tabular}


Table 4. Cont.

\begin{tabular}{ccc}
\hline Variable & $p$-Value & Normality \\
\hline ML-NSL $\left(^{\circ}\right)$ & 0.641 & Yes \\
ML-NL $\left(^{\circ}\right)$ & 0.132 & Yes \\
H & 0.803 & Yes \\
Angle $1+: 1-\left(^{\circ}\right)$ & 0.004 & No \\
Angle $1+:$ NA $\left(^{\circ}\right)$ & 0.211 & Yes \\
Angle $1-:$ NB $\left(^{\circ}\right)$ & 0.269 & Yes \\
Pg:NB $(\mathrm{mm})$ & $<0.001$ & No \\
$1+$ :NA $(\mathrm{mm})$ & $<0.001$ & No \\
$1-$ :NB $(\mathrm{mm})$ & $<0.001$ & No \\
Index & 0.972 & Yes \\
Wits $(\mathrm{mm})$ & $<0.001$ & No \\
NLA $\left(^{\circ}\right)$ & $<0.001$ & No \\
Dorsum axis N'-St $(\mathrm{mm})$ & 0.285 & Yes \\
Nose length $\mathrm{N}^{\prime}-\mathrm{Pr}(\mathrm{mm})$ & 0.47 & Yes \\
Nose depth $(1)(\mathrm{mm})$ & 0.008 & No \\
Nose depth $(2)$ Al-Pr $(\mathrm{mm})$ & 0.001 & No \\
Nose hump $(\mathrm{mm})$ & $<0.001$ & No \\
NBA $\left(^{\circ}\right)$ & 0.475 & Yes \\
NMA $\left(^{\circ}\right)$ & $<0.001$ & No \\
SFC $\left({ }^{\circ}\right)$ & $<0.001$ & No \\
Dconv & $<0.001$ & No \\
Cconv & $<0.001$ & No \\
NboneL $(m m)$ & 0.003 & No \\
NboneA $\left({ }^{\circ}\right)$ & 0.976 & Yes \\
\hline & &
\end{tabular}

Table 5. Distribution of the study group according to nasal and cephalometric values.

\begin{tabular}{|c|c|c|c|c|c|}
\hline \multicolumn{2}{|c|}{ Variable } & Females & Males & Total & $p^{1}$ \\
\hline SNA $\left(^{\circ}\right)$ & $\begin{array}{c}\text { mean } \pm S D \\
\text { median } \\
\text { quartiles }\end{array}$ & $\begin{array}{c}81.07 \pm 3.76 \\
81.1 \\
78.9-83.6\end{array}$ & $\begin{array}{c}80.13 \pm 4.71 \\
80.1 \\
77.2-82.9\end{array}$ & $\begin{array}{c}80.43 \pm 4.68 \\
80.5 \\
78.0-83.1\end{array}$ & $\begin{array}{c}0.022 \\
\text { NP }\end{array}$ \\
\hline $\mathrm{SNB}\left({ }^{\circ}\right)$ & $\begin{array}{c}\text { mean } \pm S D \\
\text { median } \\
\text { quartiles }\end{array}$ & $\begin{array}{c}78.1 \pm 3.88 \\
78.05 \\
75.38-80.53\end{array}$ & $\begin{array}{c}78.04 \pm 5.14 \\
77.8 \\
74.7-81.1\end{array}$ & $\begin{array}{c}77.94 \pm 4.71 \\
77.9 \\
74.9-80.6\end{array}$ & $\begin{array}{c}0.572 \\
\text { NP }\end{array}$ \\
\hline $\operatorname{ANB}\left({ }^{\circ}\right)$ & $\begin{array}{c}\text { mean } \pm S D \\
\text { median } \\
\text { quartiles }\end{array}$ & $\begin{array}{c}2.96 \pm 3.08 \\
3 \\
1.1-5.2\end{array}$ & $\begin{array}{c}2.09 \pm 3.7 \\
2.5 \\
-0.2-4.6\end{array}$ & $\begin{array}{c}2.49 \pm 3.5 \\
2.7 \\
0.58-4.9\end{array}$ & $\begin{array}{c}0.031 \\
\text { NP }\end{array}$ \\
\hline $\operatorname{SNPg}\left({ }^{\circ}\right)$ & $\begin{array}{c}\text { mean } \pm S D \\
\text { median } \\
\text { quartiles }\end{array}$ & $\begin{array}{c}79.09 \pm 4.06 \\
79.2 \\
76.4-81.6\end{array}$ & $\begin{array}{c}79.07 \pm 5.26 \\
78.5 \\
75.5-82\end{array}$ & $\begin{array}{c}78.97 \pm 4.82 \\
78.9 \\
75.9-81.7\end{array}$ & $\begin{array}{c}0.473 \\
\text { NP }\end{array}$ \\
\hline $\operatorname{NSBa}\left({ }^{\circ}\right)$ & $\begin{array}{c}\text { mean } \pm S D \\
\text { median } \\
\text { quartiles }\end{array}$ & $\begin{array}{c}129.81 \pm 5.4 \\
129.5 \\
126.1-133.4\end{array}$ & $\begin{array}{c}129.21 \pm 5.49 \\
129.4 \\
125-133.1\end{array}$ & $\begin{array}{c}129.5 \pm 5.52 \\
129.4 \\
125.6-133.3\end{array}$ & $\begin{array}{c}0.286 \\
\mathrm{P}\end{array}$ \\
\hline GntgoAr $\left(^{\circ}\right)$ & $\begin{array}{c}\text { mean } \pm \text { SD } \\
\text { median } \\
\text { quartiles }\end{array}$ & $\begin{array}{c}125.92 \pm 7.98 \\
125.2 \\
120.5-131.6\end{array}$ & $\begin{array}{c}127.7 \pm 7.73 \\
127.9 \\
122.4-132.8\end{array}$ & $\begin{array}{c}126.77 \pm 7.93 \\
126.20 \\
121.2-132.3\end{array}$ & $\begin{array}{c}0.03 \\
P\end{array}$ \\
\hline NL-NSL $\left(^{\circ}\right)$ & $\begin{array}{c}\text { mean } \pm \text { SD } \\
\text { median } \\
\text { quartiles }\end{array}$ & $\begin{array}{c}7.8 \pm 3.66 \\
7.7 \\
5.6-10.2\end{array}$ & $\begin{array}{c}7.89 \pm 3.88 \\
7.9 \\
5.7-10.3\end{array}$ & $\begin{array}{c}7.86 \pm 4.23 \\
7.8 \\
5.6-10.3\end{array}$ & $\begin{array}{c}0.671 \\
\text { NP. }\end{array}$ \\
\hline ML-NSL $\left(^{\circ}\right)$ & $\begin{array}{c}\text { mean } \pm S D \\
\text { median } \\
\text { quartiles }\end{array}$ & $\begin{array}{c}32.27 \pm 6.59 \\
32.3 \\
28.2-36.9\end{array}$ & $\begin{array}{c}33.6 \pm 6.85 \\
34.3 \\
28.6-38.4\end{array}$ & $\begin{array}{c}32.9 \pm 6.76 \\
32.9 \\
28.3-37.5\end{array}$ & $\begin{array}{c}0.058 \\
\mathrm{P}\end{array}$ \\
\hline
\end{tabular}


Table 5. Cont.

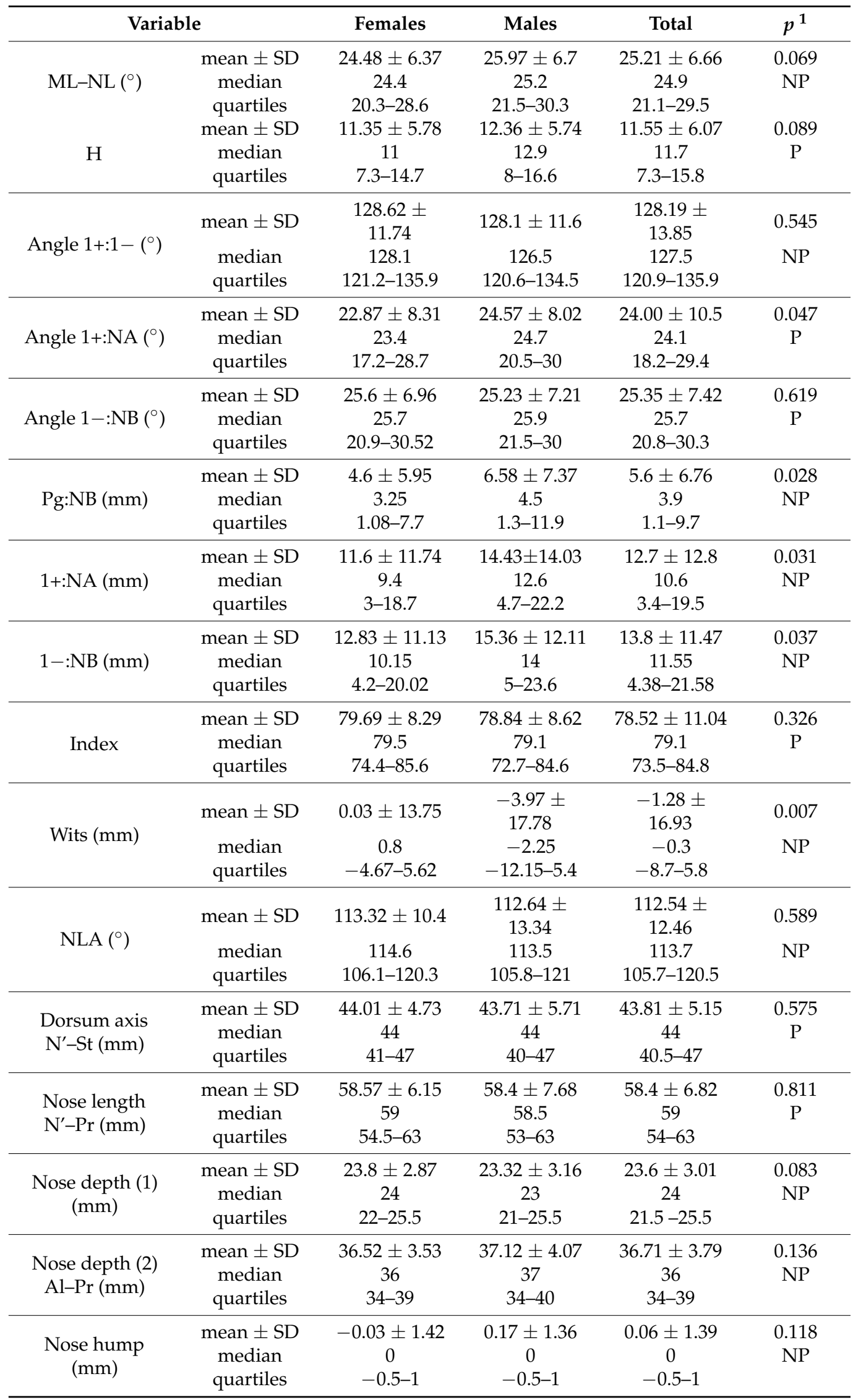


Table 5. Cont.

\begin{tabular}{|c|c|c|c|c|c|}
\hline \multicolumn{2}{|c|}{ Variable } & Females & Males & Total & $p^{1}$ \\
\hline NBA $\left(^{\circ}\right)$ & $\begin{array}{c}\text { mean } \pm S D \\
\text { median } \\
\text { quartiles }\end{array}$ & $\begin{array}{c}96.5 \pm 7.16 \\
96 \\
91.5-101\end{array}$ & $\begin{array}{c}98.04 \pm 7.71 \\
97.5 \\
93-103\end{array}$ & $\begin{array}{c}97.07 \pm 7.49 \\
97 \\
92-102\end{array}$ & $\begin{array}{c}0.046 \\
\mathrm{P}\end{array}$ \\
\hline $\operatorname{NMA}\left({ }^{\circ}\right)$ & $\begin{array}{c}\text { mean } \pm S D \\
\text { median } \\
\text { quartiles }\end{array}$ & $\begin{array}{c}122.33 \pm 6.08 \\
123 \\
118.5-126\end{array}$ & $\begin{array}{c}124.46 \pm 6.72 \\
124 \\
120-128\end{array}$ & $\begin{array}{c}123.43 \pm 6.55 \\
123.50 \\
120-127\end{array}$ & $\begin{array}{c}0.018 \\
\text { NP }\end{array}$ \\
\hline $\operatorname{SFC}\left(^{\circ}\right)$ & $\begin{array}{c}\text { mean } \pm S D \\
\text { median } \\
\text { quartiles }\end{array}$ & $\begin{array}{c}15.21 \pm 7.26 \\
15 \\
11-20\end{array}$ & $\begin{array}{c}14.79 \pm 7.92 \\
16 \\
11-20\end{array}$ & $\begin{array}{c}14.75 \pm 7.90 \\
15 \\
11-20\end{array}$ & $\begin{array}{c}0.579 \\
\text { NP }\end{array}$ \\
\hline Dconv & $\begin{array}{c}\text { mean } \pm S D \\
\text { median } \\
\text { quartiles }\end{array}$ & $\begin{array}{c}1.64 \pm 0.97 \\
2 \\
1-2\end{array}$ & $\begin{array}{c}1.54 \pm 0.92 \\
1.5 \\
1-2\end{array}$ & $\begin{array}{c}1.60 \pm 0.95 \\
1.5 \\
1-2\end{array}$ & $\begin{array}{c}0.435 \\
\text { NP }\end{array}$ \\
\hline Cconv & $\begin{array}{c}\text { mean } \pm S D \\
\text { median } \\
\text { quartiles }\end{array}$ & $\begin{array}{c}3.45 \pm 1.15 \\
3 \\
3-4\end{array}$ & $\begin{array}{c}3.67 \pm 1.28 \\
4 \\
3-4.5\end{array}$ & $\begin{array}{c}3.57 \pm 1.26 \\
3.5 \\
3-4.5\end{array}$ & $\begin{array}{c}0.098 \\
\text { NP }\end{array}$ \\
\hline NboneL (mm) & $\begin{array}{c}\text { mean } \pm S D \\
\text { median } \\
\text { quartiles }\end{array}$ & $\begin{array}{c}30.42 \pm 4.51 \\
30 \\
27.5-33\end{array}$ & $\begin{array}{c}30.84 \pm 5.3 \\
31 \\
28-34\end{array}$ & $\begin{array}{c}30.66 \pm 5.02 \\
30.50 \\
28-33.5\end{array}$ & $\begin{array}{c}0.465 \\
\text { NP }\end{array}$ \\
\hline NboneA $\left({ }^{\circ}\right)$ & $\begin{array}{c}\text { mean } \pm S D \\
\text { median } \\
\text { quartiles }\end{array}$ & $\begin{array}{c}148.02 \pm 9.58 \\
147.5 \\
142-154\end{array}$ & $\begin{array}{c}148.61 \pm \\
10.21 \\
149 \\
141-156\end{array}$ & $\begin{array}{c}148.25 \pm 9.95 \\
148.50 \\
142-155\end{array}$ & $\begin{array}{c}0.568 \\
\mathrm{P}\end{array}$ \\
\hline
\end{tabular}

${ }^{1} \mathrm{P}=$ normality of distribution in both groups, $t$-test; $\mathrm{NP}$ = lack of normality in at least one group, Mann-Whitney test.

The matrix of correlations between nasal and cephalometric variables is presented in Figure 2. The blue area shows strong positive correlation, the red area shows strong negative correlation. SFC angle shows the strongest correlations with Holdaway ratio $(\mathrm{H})$, sagittal angle between maxilla and mandible (ANB), and Wits appraisal (Wits).

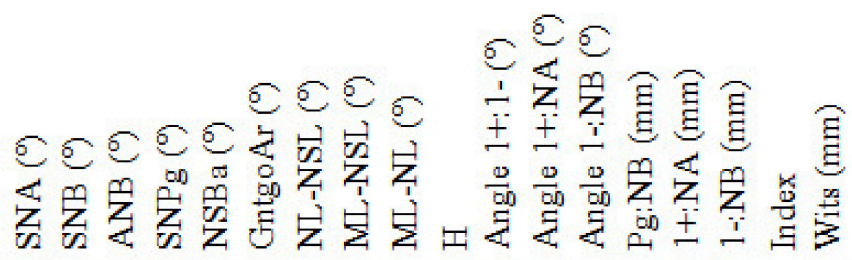

NLA $\left({ }^{\circ}\right)$

Dorsum axis N'-St (mm)

Nose length $\mathrm{N}^{\prime}-\operatorname{Pr}(\mathrm{mm})$

Nose depth (1) (mm)

Nose depth (2) Al-Pr (mm)

Nose hump (mm)

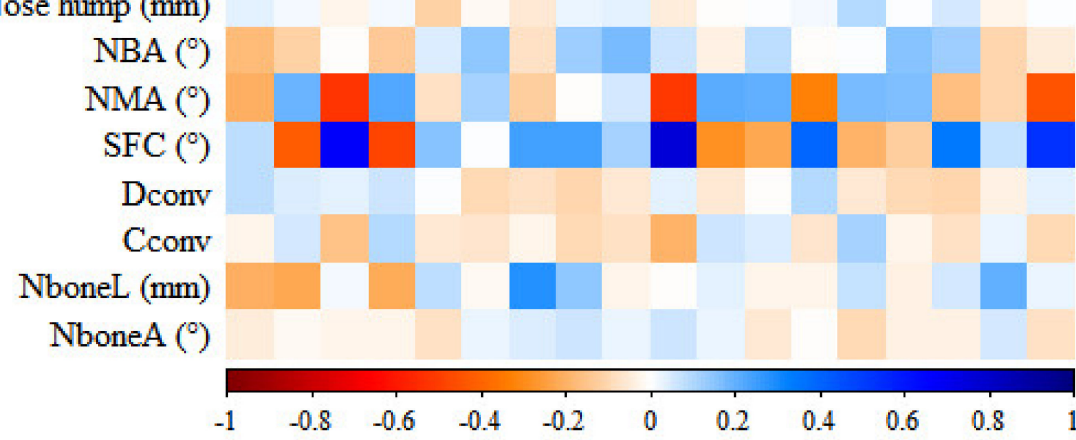

Figure 2. Correlation matrix for nasal and cephalometric variables. 
In the study group, a weak positive correlation with age was found for the following nasal parameters: dorsum axis, nose length, nose depth (1), nose depth (2), and a very weak positive for: nose hump (Hump), lower dorsum convexity (Dconv), columella convexity (Cconv). No correlation with age was stated for nasal bone length (NboneL). A weak negative correlation with age was found for NBA and a very weak negative correlation for: NLA, NMA, soft tissue facial convexity (SFC), and nasal bone angle (NboneA).

Significant differences were found between the sexes. Table 5 shows distribution of the study group according to nasal and cephalometric values referring to sex. Nasal base angle (NBA) and nasomental angle (NMA) were significantly smaller in females $(p<0.05)$. The average NLA is $113.32 \pm 10.4$ in females and $112.64 \pm 13.34$ in males, no significant differences were found between the sexes.

\section{Discussion}

Knowledge on correlations between the nasal parameters and the skeletal structures may help orthodontists and maxillofacial surgeons in diagnosing and treatment planning, for example the nose depth (1) and (2), nasal length, SFC, NMA, NBA, and hump determine the size and shape of the nose, whereas NLA angle is considered during the extraction treatment decision. The size of the patient's nose is important for maxillofacial surgeons as they influence the occlusion as well as the profile of the patient's face.

In the present study, the SFC angle showed a statistically significant positive correlation to H, ANB, and Wits, in accordance with the studies by Arshad et al. [2] as well as by Gulsen et al. [4], indicating a correlation to skeletal Classes. The SFC showed a weak negative correlation to SNB angle (Sella-Nasion to point B angle) (mandibular position), in agreement with the study by Gulsen et al. [4]. The very weak negative correlation between nasal bone length and SNA angle (maxillary position) confirms previous findings by Gulsen et al. [4] as well.

The negative correlation of the NMA angle to $\mathrm{H}, \mathrm{ANB}$, and Wits is in agreement with the results by Arshad et al. [2] as well as by Gulsen et al. [4]. The NMA angle is related to the skeletal Classes, to the upper and lower incisor inclination, to the maxillary and mandibular positions, as well as to maxillary inclination. Gulsen et al. [4] have found a significant correlation between the NMA angle and mandibular and maxillary position. In the study by Taha and Ahmed [20], the NMA angle was significantly higher in skeletal Class III compared to Class I and II.

The lack of statistically significant correlation between the Hump and skeletal Class is contrary to findings reported by Chaconas [13], who reported that Class II subjects proved to have a more pronounced nose hump, whereas Class I subjects tended to have a straighter nose [13].

The positive correlation between NLA angle and ANB angle (although very weak) is in accordance with the study by Gulsen et al. [4], but it is contrary to the results by Arshad et al. [2], as well as those by Taha and Ahmed [20]. A possible reason for this discordance may be the sizes of the study groups, e.g., insufficient number of subjects to determine statistical significance of a week correlation: Arshad et al. [2] (119 subjects), Taha and Ahmed [20] (90 subjects), Gulsen et al. [4] (262 subjects), present study (386 subjects). No significant differences concerning NLA angle were found between patients with skeletal Class I and other skeletal patterns (Class II/1, Class II/2, Class III) [21]. The very weak negative correlation between NLA and SNB (mandibular position) angles in the present study is consistent with the results by Gulsen et al. [4]. A positive correlation was found in the present investigation between NLA and mandibular inclination also increased, in accordance with the study by Gulsen et al. [4] on patients with a history of orthodontic treatment. However contrary to this are the findings by Nehra and Sharma [1], who reported no significant correlation. The reason could be that the study group was 190 Indian adults, who had undergone orthodontic treatment [1]. Burstone [8] reported that increased NLA indicated a maxillary retrusion and decreased-a maxillary protru- 
sion. Contrary conclusions have been drawn by Gulsen et al. [4], who did not find any correlations between NLA and maxillary position, similarly as the present study.

Concerning nasal growth, the dorsum shape changes, especially during adolescence (between ages 10 and 14) and the hump of the nose appears during the adolescence period and is associated with positional changes of the nasal bone $[11,22,23]$. The nasal dorsum consists of upper and lower part. The angulation of the lower part of is closely associated with vertical growth changes of the tip of the nose [11].

Nasal development is almost completed in females by the age of 16 and in males by the age of 18 [1,3,12,22,23]. However, Meng et al. [3] stated that nasal growth in males continues above the age of 18 . Nevertheless, most of the soft tissue development in women ceases at the age of 12 and in men-17 [15].

The nose is responsible for the total increase in soft tissue profile convexity with age $[12,13]$. The nose grows forward and downward and continues during maturation $[2,3,12,13,22-24]$. Compositely, this tendency increases nose prominence relatively to the facial profile [13-15,22,24-26]. However, female subjects have a smaller increase in nose depth than male subjects [3,12].

After the age of 14 years, the tip of the nose does not grow forward to the same extent as does the nasal bone and this results in nasal dorsum straightening or humping [22]. Subsequently nasal bone length is approximately $40-45$ per cent of the total length of the nose [24]. In both sexes, the increase of growth in vertical dimension is much greater than the increase in anteroposterior dimension [24]. Interestingly, it was stated, that nasal growth occurred in all patients during orthodontic therapy, including those in whom skeletal growth had declined [14]. Nasal imbalance was intensified during orthodontic treatment [14].

During growth, soft tissue facial profile, excluding the nose, tends to remain relatively stable in its degree of convexity. However, when the nose is included in profile evaluation, the convexity of the soft tissue profile increases markedly with growth $[12,15,23]$. The present study is in agreement with these findings. Angular shapes and positional relationship of the nose, lips, and chin remain relatively constant throughout the developmental period for both sexes [15].

The increase of nose depth with age found is consistent with the study by Meng et al. [3], who proved that upper and lower nose height, as well as nose depth, showed most growth between 7 and 16 years, and females are characterized by a smaller increase in nose depth. Similar results were reported by Kumar et al. [27]. Moreover, nasal growth in men continued longer, after the age of 18 [3,13].

The increase of nasal dorsum with age is in agreement with the findings by Buschang et al. [11], who reported that nasal dorsum increases 10 degrees between 6 and 14 years, and changes are slightly greater during childhood. The increase in nasal length with age is consistent with the study by Chaconas [13] and Kumar et al. [27]. It was found that nasal length was correlated to other linear cephalometric measurements changing with age, for example mandibular length $[4,13]$. The length of the nose was negatively correlated to the angular measurement $\mathrm{N}^{\prime} \operatorname{PrnP}^{\prime}$ (nasomental angle) revealing that the length of the nose contributed to the convexity of the soft tissue profile [13].

In the present study, no statistically significant difference concerning NLA was found between white men and women, which is consistent with the papers by Fitzgerald et al. [10] and Hwang et al. [28]. Similar results were reported by Bagwan et al. [29], referring to Egyptian adults. However, contrary results were found by Magnani et al. [30] in young Brazilian black from 10 to 14 years, as well as by Taha and Ahmed [20] in Iraqi adults. Moreover, statistically significant sexual dimorphism of NMA angle is contrary to the studies by Taha and Ahmed [20], Hwang et al. [28], as well as by Lopatiene et al. [31], Aljabaa [32], and Kumar et al. [27]. The different findings may result from various sizes and age ranges of the groups analyzed. Taha and Ahmed [20] reported on 45 men and 45 women (age range: 18-25 years), Hwang et al. [28] reported on 15 men and 27 women (age range: 18-34 years), whereas Lopatiene et al. [31]—on 114 patients (age range: 14-16 years), 
Aljabaa [32]—on 32 females and 30 males (age range: 20-24 years), Kumar et al. [27] reported on 80 females and 80 males (age range: 8-16 years), while the present study reports on 229 females and 157 males (age range: 9-25 years).

The results of the present study referring to significant sex differences in nasal length, nose depth (1) and nose depth (2) and nasal hump are contrary to those reported by Gulsen et al. [4] on 262 Anatolian Turkish adults (age range: 18-30 years) as well as Arshad et al. [2] on 119 subjects of Pakistani origin (age range: 18-40 years). The present study is in accordance with the study by Aljabaa [32] on 62 Saudi subjects, (age range: 20-24 years), who found statistically significant sex differences in nasal length. The present study is contrary to this by Kumar et al. [27], who did not find significant differences in nose depth. Taha and Ahmed [20] observed no significant differences concerning nasal length and nose depth between the sexes. The significant difference for SFC angle between the sexes in the present study confirms the results by Gulsen et al. [4], however, is contrary to the study by Arshad et al. [2]. The different findings may result from age structure of the study groups or racial differences. The lack of significant sexual dimorphism referring to SFC angle is in accordance with the findings by Bagwan et al. [29].

Ethnic variability should always be taken under consideration. The NLA angle is smaller in Brazilian subjects of color than in white individuals [30]. It was also proved that this angle was significantly smaller in females [30]. However, black individuals showed similar angular measurements in both sexes [10].

NLA value in the literature has been presented in Table 6. All ethnic groups, presented in Table 6, had similar NLA angle in men and woman.

Table 6. NLA value in the literature.

\begin{tabular}{|c|c|c|c|c|}
\hline \multirow{2}{*}{ Author, Year } & \multirow{2}{*}{ Population } & \multicolumn{3}{|c|}{ NLA Values } \\
\hline & & Men & Women & Total \\
\hline Burstone [33], 1967 & Caucasian adolescent & no data & no data & $74+/-8.00$ \\
\hline Fitzgerald et al. [10], 1992 & white Americans & $113.55+/-9.44$ & 116.19 & $114.08+/-9.58$ \\
\hline Arnett et al. [34], 1999 & white Americans & $106.40+/-7.70$ & $103.50+/-6.80$ & no data \\
\hline $\begin{array}{l}\text { Fernandez-Riveiro et al. [35], } \\
2003\end{array}$ & $\begin{array}{c}\text { Caucasians from Galicia, } \\
\text { young adults }\end{array}$ & $105+/-13.28$ & $107+/-8.50$ & no data \\
\hline Hwang et al. [28], 2002 & $\begin{array}{c}\text { European-American origin } \\
\text { adults }\end{array}$ & $112.05+/-9.86$ & $109.71+/-7.60$ & no data \\
\hline Hwang et al. [28], 2002 & Korean origin adults & $91.11+/-8.12$ & $92.00+/-9.55$ & no data \\
\hline Magnani et al. [30], 2004 & Young Brazilian subjects of color & $92.00+/-12.52$ & $85.05+/-11.93$ & $88.14+/-12.52$ \\
\hline Scavone et al. [36], 2006 & Japanese-Brazilian adults & $108.40+/-10.76$ & $110.10+/-8.97$ & no data \\
\hline Nehra and Sharma [1], 2009 & Indian adults & no data & no data & $92.69+/-11.09$ \\
\hline Dua et al. [37], 2010 & Indian adults population & $96.74+/-10.89$ & $95.64+/-8.90$ & $96.10+/-9.70$ \\
\hline Anic-Milosevic et al. [38], 2011 & Croatians & $105.00+/-9.52$ & $109.39+/-7.84$ & no data \\
\hline Kandhasamy et al. [39], 2012 & $\begin{array}{l}\text { Komarapalayam adults } \\
\text { population }\end{array}$ & $116.51+/-8.01$ & $115.701+/-4.00$ & $116.10+/-10.00$ \\
\hline Arshad et al. [2], 2013 & Pakistani origin & $100.55+/-14.52$ & $98.87+/-15.76$ & no data \\
\hline $\begin{array}{c}\text { Paradowska-Stolarz and } \\
\text { Kawala [40], } 2015\end{array}$ & $\begin{array}{l}\text { white adult Poles-healthy } \\
\text { patients }\end{array}$ & $116.60+/-11.53$ & $112.77+/-13.17$ & no data \\
\hline $\begin{array}{l}\text { Paradowska-Stolarz and } \\
\text { Kawala [40], } 2015\end{array}$ & $\begin{array}{l}\text { white adult Poles with any cleft } \\
\text { (lip/ palate/both) }\end{array}$ & $100.36+/-18.13$ & $101.14+/-17.51$ & no data \\
\hline Bagwan et al. [29], 2015 & Egyptian adults & $94.40+/-10.23$ & $96.46+/-11.30$ & $95.00+/-10.40$ \\
\hline Aljabaa [32], 2019 & Saudi adults & $96.23+/-12.74$ & $104.19+/-11.92$ & no data \\
\hline Taha and Ahmed [20], 2020 & Iraqi adults, skeletal Class I & $97.93+/-9.75$ & $101.73+/-12.15$ & no data \\
\hline Taha and Ahmed [20], 2020 & Iraqi adults, skeletal Class II & $91.20+/-14.95$ & $98.80+/-13.10$ & no data \\
\hline Taha and Ahmed [20], 2020 & Iraqi adults, skeletal Class III & $95.33+/-10.75$ & $101.93+/-9.81$ & no data \\
\hline Perović et al. [21], 2020 & $\begin{array}{c}\text { Caucasian adults from Serbia, } \\
\text { skeletal Class I }\end{array}$ & no data & no data & $111.67+/-10.76$ \\
\hline Present study & white adult Poles & $112.64+/-13.34$ & $113.32+/-10.40$ & $112.54+/-12.46$ \\
\hline
\end{tabular}




\section{Conclusions}

1. A relationship exists between nasal morphology and sagittal jaw configuration.

2. The following nasal parameters increase with age: dorsum axis, nose length, nose depth (1), nose depth (2), nasal hump, lower dorsum convexity, columella convexity. Parameters decreasing with age are: nasal bone angle, nasolabial angle, nasomental angle, soft tissue facial convexity, and nasal bone angle.

3. Sexual dimorphism has been found for nasal parameters: nasal bone angle and nasomental angle are significantly smaller in females.

Author Contributions: Conceptualization, A.J.; methodology, A.J. and J.J.-O.; software, A.J.; validation, A.J. and J.J.-O.; formal analysis, A.J. and J.J.-O.; investigation, A.J.; resources, A.J. and J.J.-O.; data curation, A.J.; writing—original draft preparation, A.J. and J.J.-O.; writing—review and editing, A.J., J.J.-O. and K.G.; visualization, A.J.; supervision, J.J.-O.; project administration, J.J.-O.; funding acquisition, K.G. All authors have read and agreed to the published version of the manuscript.

Funding: No funding was needed for the research. APC was founded by Pomeranian Medical University in Szczecin.

Institutional Review Board Statement: Not applicable.

Informed Consent Statement: Not applicable.

Data Availability Statement: All data is a part of the manuscript.

Conflicts of Interest: The authors declare no conflict of interests.

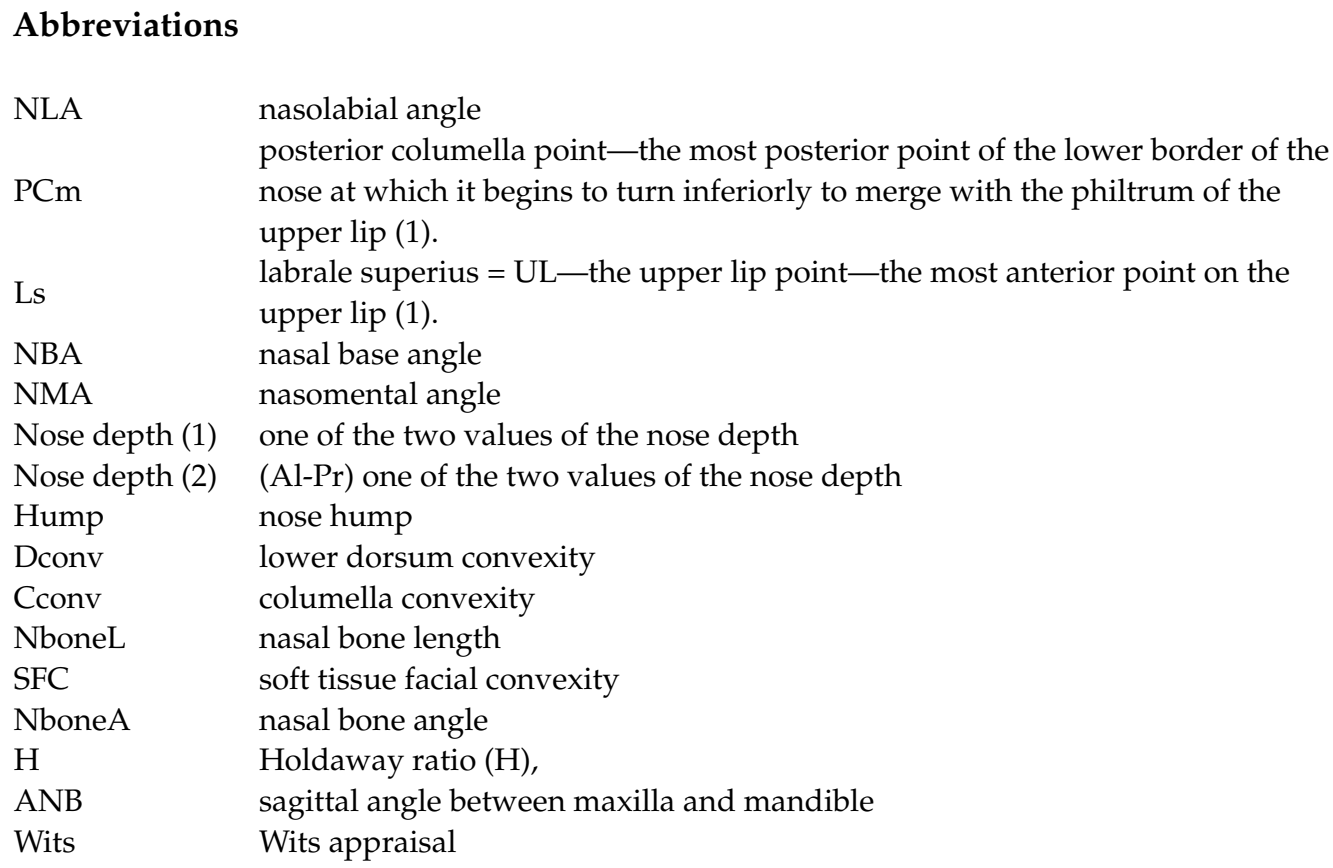

\section{References}

1. Nehra, K.; Sharma, V. Nasal morphology as an indicator of vertical maxillary skeletal pattern. J. Orthod. 2009, 36, 160-166. [CrossRef] [PubMed]

2. Arshad, T.; Shaikh, A.; Fida, M. Comparison of nasal profiles in various skeletal patterns. J. Ayub Med. Coll. Abbottabad 2013, 25, 31-35. [PubMed]

3. Meng, H.P.; Goorhuis, J.; Kapila, S.; Nanda, R.S. Growth changes in the nasal profile from 7 to 18 years of age. Am. J. Orthod. Dentofac. Orthop. 1988, 94, 317-326. [CrossRef]

4. Gulsen, A.; Okay, C.; Aslan, B.I.; Unerk, O.; Yavuzer, R. The relationship between craniofacial structures and the nose in Anatolian Turkish adults: A cephalometric evaluation. Am. J. Orthod. Dentofac. Orthop. 2006, 130, 131.e15-132.e25. [CrossRef]

5. Farkas, L.G.; Kolar, J.C.; Munro, I.R. Geography of the nose: A morphometric study. Aesthetic Plast. Surg. 1986, 10, 191-223. [CrossRef] 
6. Robison, J.M.; Rinchuse, D.J.; Zullo, T.G. Relationship of skeletal pattern and nasal form. Am. J. Orthod. 1986, 89, 499-506. [CrossRef]

7. Sarver, D.M.; Rousso, D.R. Surgical procedures to improve esthetics when orthognathic surgery is not an option. Am. J. Orthod. Dentofac. Orthop. 2004, 126, 299-301. [CrossRef]

8. Burstone, C.J. The integumental profile. Am. J. Orthod. 1958, 44, 1-25. [CrossRef]

9. Lo, F.D.; Hunter, W.S. Changes in nasolabial angle related to maxillary incisor retraction. Am. J. Orthod. 1982, 82, 384-391. [CrossRef]

10. Fitzgerald, J.P.; Nanda, R.S.; Currier, G.F. An evaluation of the nasolabial angle and the relative inclinations of the nose and upper lip. Am. J. Orthod. Dentofac. Orthop. 1992, 102, 328-334. [CrossRef]

11. Buschang, P.H.; De La Cruz, R.; Viazis, A.D.; Demirjian, A. A longitudinal shape changes of the nasal dorsum. Am. J. Orthod. Dentofac. Orthop. 1993, 104, 539-543. [CrossRef]

12. Chaconas, S.J.; Bartroff, J.D. Prediction of normal soft tissue facial changes. Angle Orthod. 1975, 45, 12-25. [CrossRef] [PubMed]

13. Chaconas, S.J. A statistical evaluation of nasal growth. Am. J. Orthod. 1969, 56, 403-414. [CrossRef]

14. Clements, B.S. Nasal imbalance and the orthodontic patient. Am. J. Orthod. 1969, 55, 477-498. [CrossRef]

15. Genecov, J.S.; Sinclair, P.M.; Dechow, P.C. Development of the nose and soft tissue profile. Angle Orthod. 1990, 60, 191-198. [CrossRef] [PubMed]

16. Garner, L.D. Soft tissue changes concurrent with orthodontie tooth movement. Am. J. Orthod. 1974, 66, 367-377. [CrossRef]

17. Segner, D.; Hasund, A. Individualisierte Kephalometrie, 4th ed.; Dietmar Segner Verlag: Hamburg, Germany, 2003 ; pp. 7-27.

18. R Core Team. R: A Language and Environment for Statistical Computing; R Foundation for Statistical Computing: Vienna, Austria, 2020; Available online: https:/ / www.r-project.org/ (accessed on 2 August 2020).

19. Yadav, S. Correlation analysis in biological studies. J. Pract. Cardiovasc. Sci. 2018, 4, 116-121. [CrossRef]

20. Taha, A.A.; Ahmed, A.S. A comprehensive assessment of the nasal profile among Iraqi adults with different skeletal classes: A retrospective study. J. Oral Biol. Craniofac. Res. 2020, 10, 175-179. [CrossRef]

21. Perović, T.M.; Blažej, Z.; Jovanović, I. The influence of antero-posterior dentoskeletal pattern on the value of nasal soft tissue angles: A cephalometric study. Folia Morphol. 2020. [CrossRef] [PubMed]

22. Posen, J.M. A longitudinal study of the growth of the nose. Am. J. Orthod. 1967, 53, 746-756. [CrossRef]

23. Subtelny, J.D. A longitudinal study of soft tissue facial structures and their profile characteristics, defined in relation to underlying skeletal structures. Am. J. Orthod. 1959, 45, 481-507. [CrossRef]

24. Manera, J.F.; Subtelny, J.D. A cephalometric study of the growth of the nose. Am. J. Orthod. 1961, 47, 703-705.

25. Clements, B.S. Nasal imbalance and the orthodontic patient. Am. J. Orthod. 1969, 55, 244-264. [CrossRef]

26. Clements, B.S. Nasal imbalance and the orthodontic patient. Am. J. Orthod. 1969, 55, 329-352. [CrossRef]

27. Kumar, A.; Tandon, P.; Singh, G.K.; Singh, G.P. Soft tissue growth changes from 8 to 16 years of age: A cross-sectional study. Natl. J. Maxillofac. Surg. 2019, 10, 161-167. [CrossRef] [PubMed]

28. Hwang, H.S.; Kim, W.S.; McNamara, J.A., Jr. Ethnic differences in Soft Tissue Profile of Korean and European-American adults with normal occlusions and well balanced faces. Angle Orthod. 2002, 72, 72-80. [CrossRef] [PubMed]

29. Bagwan, A.A.; Alshennawy, M.; Alskhawy, M.M. Evaluation of soft tissue parameters for adults with accepted occlusion using Legan and Burstone analysis. Tanta Dent. J. 2015, 12, 1-6. [CrossRef]

30. Magnani, M.B.; Nouer, D.F.; Nouer, P.R.; Pereira Neto, J.S.; Garbui, I.U.; Böeck, E.M. Assessment of the nasolabial angle in young Brazilian black subjects with normal occlusion. Braz. Oral Res. 2004, 18, 233-237. [CrossRef]

31. Lopatienè, K.; Šidlauskas, A.; Vasiliauskas, A.; Čečytè, L.; Švalkauskienė, V.; Šidlauskas, M. Relationship between malocclusion, soft tissue profile, and pharyngeal airways: A cephalometric study. Medicina 2016, 52, 307-314. [CrossRef] [PubMed]

32. Aljabaa, A.H. Lateral cephalometric analysis of the nasal morphology among Saudi adults. Clin. Cosmet. Investig. Dent. 2019, 11, 9-17. [CrossRef]

33. Burstone, C.J. Lip posture and its significance in treatment planning. Am. J. Orthod. 1967, 53, 262-284. [CrossRef]

34. Arnett, G.W.; Jelic, J.S.; Kim, J.; Cummings, D.R.; Beress, A.; Worley, C.M., Jr.; Chung, B.; Bergman, R. Soft tissue cephalometric analysis: Diagnosis and treatment planning of dentofacial deformity. Am. J. Orthod. Dentofac. Orthop. 1999, 116, $239-253$. [CrossRef]

35. Fernández-Riveiro, P.; Smyth-Chamosa, E.; Suárez-Quintanilla, D.; Suárez-Cunqueiro, M. Angular photogrammametric analysis of soft tissue profile. Eur. J. Orthod. 2003, 25, 393-399. [CrossRef] [PubMed]

36. Scavone, H., Jr.; Trevisan, H., Jr.; Garib, D.G.; Ferreira, F.V. Facial profile evaluation in Japanese-Brazilian adults with normal occlusions and well balanced faces. Am. J. Orthod. Dentofac. Orthop. 2006, 129, 721.e1-721.e5. [CrossRef] [PubMed]

37. Dua, V.; Gupta, S.; Singh, C. Evaluation of the nasolabial angle in the Indian population. Contemp. Clin. Dent. 2010, 1, 79-82. [CrossRef] [PubMed]

38. Anić-Milošević, S.; Meštrović, S.; Lapter-Varga, M.; Dumancic, J.; Šlaj, M. Analysis of soft tissue profile in Croatians with normal occlusions and well-balanced faces. Eur. J. Orthod. 2011, 33, 305-310. [CrossRef] [PubMed]

39. Kandhasamy, K.; Prabu, N.M.; Sivanmalai, S.; Prabu, P.S.; Philip, A.; Chiramel, J.C. Evaluation of the nasolabial angle of the Komarapalayam population. J. Pharm. Bioallied Sci. 2012, 4, S313-S315. [CrossRef]

40. Paradowska-Stolarz, A.M.; Kawala, B. The Nasolabial Angle Among Patients with Total Cleft Lip and Palate. Adv. Clin. Exp. Med. 2015, 24, 481-485. [CrossRef] [PubMed] 